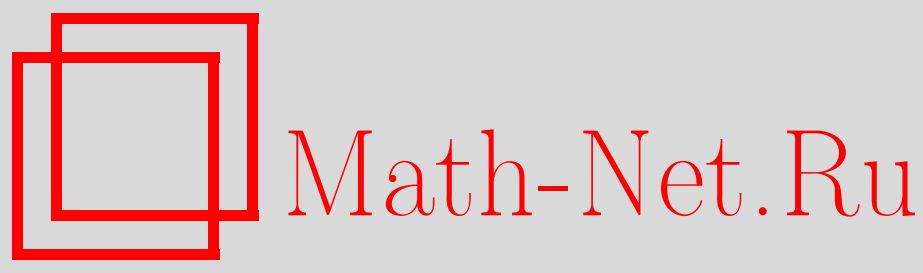

В. В. Вальков, А. О. Злотников, Сосуществование сверхпроводимости и антиферромагнетизма в тяжелофермионных интерметаллидах, ТМФ, 2013, том 174, номер 3, 484-503

DOI: https://doi.org/10.4213/tmf8378

Использование Общероссийского математического портала Math-Net.Ru подразумевает, что вы прочитали и согласны с пользовательским соглашением http://www.mathnet.ru/rus/agreement

Параметры загрузки:

IP : 18.234 .197 .8

26 апреля 2023 г., 14:29:12

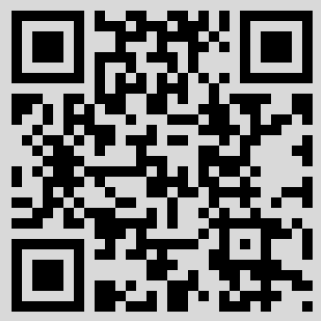




\title{
СОСУЩЕСТВОВАНИЕ СВЕРХПРОВОДИМОСТИ И АНТИФЕРРОМАГНЕТИЗМА В ТЯЖЕЛОФЕРМИОННЫХ ИНТЕРМЕТАЛЛИДАХ
}

\begin{abstract}
В рамках эффективного гамильтониана для периодической модели Андерсона при использовании двухвременных запаздывающих функций Грина изучены условия реализации фазы сосуществования сверхпроводимости и антиферромагнетизма. Такая фаза экспериментально наблюдается в редкоземельных интерметаллидах с тяжелыми фермионами при приложении внешнего давления. В выбранной модели при наличии дальнего антиферромагнитного упорядочения куперовская неустойчивость индуцируется в результате совместного влияния сверхобменного взаимодействия в подсистеме локализованных электронов и гибридизации между двумя группами электронов. Приложение внешнего давления вызывает увеличение энергии локализованного уровня, сопровождающееся в определенной области фазовой диаграммы резким разрушением дальнего антиферромагнитного упорядочения. В точке разрушения сверхпроводящий параметр порядка обладает максимальным значением. Показано, что уменьшение намагниченности антиферромагнитной подрешетки с ростом давления вызывает значительное увеличение массы фермиевских квазичастиц, а в критической точке происходит изменение знака носителей тока. Полученные результаты на качественном уровне хорошо согласуются с экспериментальными данными для тяжелофермионного интерметаллида $\mathrm{CeRhIn}$.
\end{abstract}

Ключевые слова: периодическая модель Андерсона, сосуществование сверхпроводимости и антиферромагнетизма, сверхобменное взаимодействие, тяжелые фермионы.

DOI: $10.4213 / \operatorname{tmf} 8378$

\section{1. ВВЕДЕНИЕ}

Известно, что сильные электронные корреляции в существенной степени определяют свойства слабо легированных оксидов меди [1], [2] и тяжелофермионных (ТФ) интерметаллидов [3]-[6]. В этих материалах реализуется нефононный механизм

*Федеральное государственное бюджетное учреждение науки Институт физики им. Л. В. Киренского СО РАН, Красноярск, Россия.

E-mail: vvv@iph.krasn.ru, zlotn@iph.krasn.ru

${ }^{\dagger}$ Сибирский государственный аэрокосмический университет им. М. Ф. Решетнёва, Красноярск, Россия 
сверхпроводящего спаривания [1], [7]-[9], и куперовская неустойчивость развивается либо на фоне разрушенного антиферромагнитного упорядочения при сохранении ближних антиферромагнитных корреляций, либо при наличии дальнего антиферромагнитного порядка, но с существенно уменьшенным значением намагниченности подрешетки.

Реализация микроскопически однородной фазы сосуществования сверхпроводимости (СП) и антиферромагнетизма (АФМ) в сильно коррелированных системах в последнее время стала предметом повышенного внимания. Это произошло благодаря тому, что приложение внешнего давления к ряду ТФ-антиферромагнетиков приводит к индуцированию фазового перехода с формированием отмеченной фазы сосуществования.

Известно, что в проводящих антиферромагнетиках условия реализации куперовской неустойчивости являются более благоприятными, чем в ферромагнитных металлах [10], [11]. Это объясняется отсутствием спонтанной макроскопической намагниченности и сохранением вырожденности спектра фермиевских возбуждений по направлению проекции спинового момента. Примеров соединений, в которых наблюдается фаза сосуществования СП и АФМ, известно немало. Впервые явление одновременной реализации СП и АФМ в ТФ-системах было зафиксировано в трансурановых соединениях $\mathrm{UPt}_{3}$ [12], $\mathrm{UPd}_{2} \mathrm{Al}_{3}$ [13]. В этих соединениях два из трех 5 -электронов урана являются локализованными и отвечают за индуцирование антиферромагнитного упорядочения [14]. Третий 5f-электрон участвует в формировании зоны тяжелых фермионов. В ансамбле таких электронов развивается куперовская неустойчивость, приводящая к реализации сверхпроводящей ТФ-фазы.

В отличие от трансурановых материалов в цериевом интерметаллиде $\mathrm{CeRhIn}_{5}$, относящемся к группе $\mathrm{Ce}_{n} \mathrm{~T}_{m} \mathrm{In}_{3 n+2 m}$ (здесь $\mathrm{T}=\mathrm{Co}, \mathrm{Rh}, \mathrm{Pd}, \mathrm{Ir}, \mathrm{Pt} ; n=1,2 ; m=0,1$ ), 4f-электрон церия принимает участие в формировании как дальнего антиферромагнитного порядка, так и куперовской неустойчивости. На это косвенно указывает совпадение энтропии при переходе из антиферромагнитной фазы в сверхпроводящую фазу под действием внешнего давления [15]. При этом вблизи такого перехода реализуется область, в которой СП и АФМ сосуществуют на микроскопическом уровне [16]. Фаза сосуществования ограничена диапазоном давлений $P \approx 1 \div 1.8$ ГПа с максимальной критической температурой около 2 K [15].

Сильная взаимосвязь между СП и АФМ была установлена также в оксидах меди [17]. Посредством измерения сдвига Найта методом ядерного магнитного резонанса было продемонстрировано сосуществование высокотемпературной СП и антиферромагнитного порядка в многослойных купратах $\mathrm{HgBa}_{2} \mathrm{Ca}_{4} \mathrm{Cu}_{5} \mathrm{O}_{12+\delta}$ [18] и $\mathrm{Ba}_{2} \mathrm{Ca}_{n-1} \mathrm{Cu}_{n} \mathrm{O}_{2 n}(\mathrm{~F}, \mathrm{O})_{2}(n=4,5)$ [19]. Сосуществование фаз в двухслойном меднооксидном сверхпроводнике $\mathrm{YBa}_{2} \mathrm{Cu}_{3} \mathrm{O}_{6+x}$ было показано в работе [20].

В связи со сказанным представляется актуальным проведение теоретического анализа, направленного на изучение нефононного механизма куперовской неустойчивости, который приводит к формированию на микроскопическом уровне однородной сверхпроводящей фазы с $d$-типом симметрии параметра порядка при наличии дальнего антиферромагнитного упорядочения. В качестве сопутствующей цели такого исследования может выступать задача интерпретации аномального поведения эффективной массы в окрестности квантового фазового перехода.

Целесообразно подчеркнуть, что формирование фазы сосуществования СП и АФМ может развиваться по двум сценариям. Согласно первому из них за реализацию СП 
и АФМ ответственны две разные группы электронов: СП устанавливается в подсистеме коллективизированных фермионов, тогда как дальний антиферромагнитный порядок реализуется благодаря наличию локализованных электронов. Этот сценарий имеет место в тройных редкоземельных [21] и в урановых ТФ-соединениях.

Второй сценарий образования фазы сосуществования проявляется в цериевых интерметаллидах. В них за формирование обоих типов упорядочения ответствен-

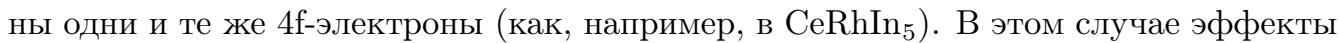
взаимного влияния СП и АФМ играют более существенную роль и являются предметом многих теоретических исследований. В работе [22] фаза сосуществования СП и АФМ исследовалась на основе среднеполевого слейв-бозонного приближения в рамках периодической модели Андерсона (ПМА), которая дополнялась квадратичными по фермиевским операторам слагаемыми, описывающими как куперовскую неустойчивость, так и неустойчивость по отношению к антиферромагнитному состоянию. Следует отметить, что такой подход оставлял открытым вопрос о виде электронного взаимодействия в ТФ-системах, приводящего к смешанному состоянию, в котором одновременно реализуются сверхпроводящие и антиферромагнитные свойства. В работе [22] область сосуществования СП и АФМ была получена путем увеличения величины гибридизационного параметра и ширины зоны электронов. Увеличение этих параметров связывалось с ростом внешнего гидростатического давления. Более простое рассмотрение СП и АФМ в системах с тяжелыми фермионами было реализовано в работе [23]. Исследовался ансамбль коллективизированных электронов, которые взаимодействуют между собой посредством одноузельного кулоновского отталкивания и эффективного взаимодействия, приводящего к притяжению. Для получения тяжелой эффективной массы электронов были введены дополнительные ограничения на модель. Полагалось, что уровень Ферми лежит в окрестности седловой точки (точки $X$ двумерной зоны Бриллюэна) и вводилось дополнительное предположение о наличии логарифмической особенности ван Хова вблизи химического потенциала.

В настоящей работе на основе последовательного применения к расширенной модели Андерсона метода двухвременных температурных функций в атомном представлении проанализированы условия, при которых становится возможным сосуществование СП и АФМ в сильно коррелированных системах с тяжелыми фермионами. В рамках такого подхода нет необходимости использовать переход к слейв-бозонам и вводить приближенное описание для учета констрейна. При этом удается получить замкнутую самосогласованную систему уравнений, описывающую как все необходимые фазы, включая фазу сосуществования, так и аномальное поведение эффективной массы фермиевских квазичастиц в окрестности квантового фазового перехода.

\section{2. ГАМИЛЬТОНИАН ЭЛЕКТРОННОЙ СТРУКТУРЫ ТЯЖЕЛОФЕРМИОННЫХ ИНТЕРМЕТАЛЛИДОВ}

Описание свойств редкоземельных интерметаллидов с тяжелыми фермионами обычно проводится в рамках ПМА [24]-[26]. В режиме сильных электронных корреляций, когда параметр $U$ кулоновского отталкивания двух локализованных электронов с противоположными проекциями спинового момента, находящихся на одном узле, существенно превышает все другие характерные энергии, локализованное состояние характеризуется однократным электронным заполнением. В этом случае 
естественным описанием является атомное представление, в явной форме учитывающее эту особенность [27]. При этом учет процессов, отражающих виртуальные переходы локализованных состояний в двухэлектронный сектор гильбертова пространства, приводит к эффективному гамильтониану, содержащему ряд дополнительных слагаемых [28]. Так, например, возникают существенные для дальнейшего слагаемые, которые описывают обменное взаимодействие в подсистеме локализованных электронов. Актуальность учета этого взаимодействия обусловлена прежде всего тем, что оно способно индуцировать как антиферромагнитное упорядочение, так и куперовскую неустойчивость. Например, в работе [29] было показано, что обменное взаимодействие в ансамбле коллективизированных электронов может приводить к реализации смешанной фазы.

В представлении Ванье эффективный гамильтониан ПМА может быть записан в виде

$$
\begin{aligned}
\widehat{\mathscr{H}}_{\mathrm{eff}}= & \sum_{m, \sigma}\left(\varepsilon_{0}-\mu\right) c_{m \sigma}^{\dagger} c_{m \sigma}+\sum_{m, l, \sigma} t_{m l} c_{m \sigma}^{\dagger} c_{l \sigma}+\sum_{m, \sigma}\left(E_{0}-\mu\right) X_{m}^{\sigma \sigma}+ \\
& \left.+\sum_{m, l, \sigma}\left\{\left(V_{m l} c_{m \sigma}^{\dagger} X_{l}^{0 \sigma}\right)+\text { (э.c. }\right)\right\}+\frac{1}{2} \sum_{m \neq l} J_{m l}\left(\vec{S}_{m} \vec{S}_{l}-\frac{1}{4} \widehat{N}_{m} \widehat{N}_{l}\right),
\end{aligned}
$$

где $c_{m \sigma}\left(c_{m \sigma}^{\dagger}\right)$ - фермиевский оператор уничтожения (рождения) коллективизированного электрона для ячейки Ванье с номером $m$ и проекцией спинового момента $\sigma$, $X_{m}^{r t}$ - оператор Хаббарда, относящийся к ячейке $m$, который строится с помощью атомных состояний $|m ; r\rangle$ и $|m ; t\rangle$ обычным образом: $X_{m}^{r t}=|m ; r\rangle\langle t ; m| ; \vec{S}_{m}$ - квазиспиновый векторный оператор локализованной подсистемы, компоненты которого связаны с операторами атомного представления формулами

$$
S_{m}^{x}=\frac{X_{m}^{\uparrow \downarrow}+X_{m}^{\downarrow \uparrow}}{2}, \quad S_{m}^{y}=-i \frac{X_{m}^{\uparrow \downarrow}-X_{m}^{\downarrow \uparrow}}{2}, \quad S_{m}^{z}=\frac{X_{m}^{\uparrow \uparrow}-X_{m}^{\downarrow \downarrow}}{2} .
$$

Оператор числа локализованных электронов на узле $m$ определяется формулой $\widehat{N}_{m}=\sum_{\sigma} X_{m}^{\sigma \sigma}$. Параметрами модели являются $\varepsilon_{0}$ - одноузельная энергия коллективизированного электрона, $\mu$ - химический потенциал, $t_{m l}$ - матричный элемент перескоков коллективизированных электронов с узла $l$ на узел $m$, а также $E_{0}-$ положение энергетического уровня локализованных электронов, $V_{m l}$ - матричный элемент, который описывает гибридизацию локализованного и коллективизированного состояний, относящихся к одной ячейке Ванье $(m=l)$ или к разным ячейкам $(m \neq l)$, и $J_{m l}-$ интеграл обменного взаимодействия.

Предполагая возможность антиферромагнитного упорядочения, перейдем к двухподрешеточному описанию. С этой целью, как обычно, выделим два типа узлов решетки. K первому типу (подрешетке $F$ ) отнесем узлы, нумеруемые в дальнейшем индексами $f, f^{\prime}, f^{\prime \prime}, \ldots$, для которых при наличии дальнего антиферромагнитного порядка $\left\langle S_{f}^{z}\right\rangle>0$. Узлы второго типа (подрешетка $G$ ) будут нумероваться индексами $g, g^{\prime}, g^{\prime \prime}, \ldots$; для узлов этой подрешетки $\left\langle S_{g}^{z}\right\rangle<0$.

В двухподрешеточном представлении гамильтониан удобно записать как

$$
\widehat{\mathscr{H}}_{\text {eff }}=\widehat{\mathscr{H}}_{0}+\widehat{\mathscr{H}}_{\text {mix }}+\widehat{\mathscr{H}}_{\text {exch }},
$$

где $\widehat{\mathscr{H}}_{0}$ описывает систему невзаимодействующих локализованных и коллективизированных электронов; с помощью $\mathscr{\mathscr { H }}_{\mathrm{mix}}$ учитываются гибридизационные процессы 
между локализованными и коллективизированными состояниями как в пределах

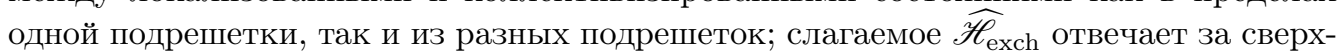
обменное взаимодействие в локализованной подсистеме. Конкретный вид этих операторов определяется выражениями

$$
\begin{aligned}
\widehat{\mathscr{H}}_{0}= & \sum_{f, \sigma}\left(\varepsilon_{0}-\mu\right) a_{f \sigma}^{\dagger} a_{f \sigma}+\sum_{g, \sigma}\left(\varepsilon_{0}-\mu\right) b_{g \sigma}^{\dagger} b_{g \sigma}+\sum_{f, \sigma}\left(E_{0}-\mu\right) X_{f}^{\sigma \sigma}+ \\
& +\sum_{g, \sigma}\left(E_{0}-\mu\right) Y_{g}^{\sigma \sigma}+\sum_{f, f^{\prime}, \sigma} t_{f f^{\prime}} a_{f \sigma}^{\dagger} a_{f^{\prime} \sigma}+\sum_{g, g^{\prime}, \sigma} t_{g g^{\prime}} b_{g \sigma}^{\dagger} b_{g^{\prime} \sigma}+ \\
& +\sum_{f, g, \sigma}\left(t_{f g} a_{f \sigma}^{\dagger} b_{g \sigma}+t_{g f} b_{g \sigma}^{\dagger} a_{f \sigma}\right), \\
\widehat{\mathscr{H}}_{\text {mix }}= & \sum_{f, f^{\prime}, \sigma}\left\{\left(V_{f f^{\prime}} a_{f \sigma}^{\dagger} X_{f^{\prime}}^{0 \sigma}\right)+(\text { э.c. })\right\}+\sum_{g, g^{\prime}, \sigma}\left\{\left(V_{g g^{\prime}} b_{g \sigma}^{\dagger} Y_{g^{\prime}}^{0 \sigma}\right)+(\text { э.c. })\right\}+ \\
& +\sum_{f, g, \sigma}\left\{\left(W_{f g} a_{f \sigma}^{\dagger} Y_{g}^{0 \sigma}+W_{g f} b_{g \sigma}^{\dagger} X_{f}^{0 \sigma}\right)+(\text { э.c. })\right\}, \\
\widehat{\mathscr{H}}_{\mathrm{exch}}= & \frac{1}{2} \sum_{\langle f g\rangle, \sigma} J_{f g}\left(X_{f}^{\sigma \bar{\sigma}} Y_{g}^{\bar{\sigma} \sigma}-X_{f}^{\sigma \sigma} Y_{g}^{\bar{\sigma} \bar{\sigma}}\right) .
\end{aligned}
$$

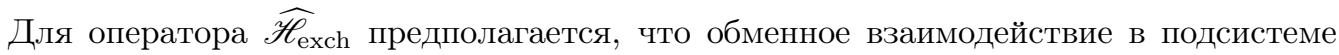
квазилокализованных состояний реализуется только между ближайшими соседями.

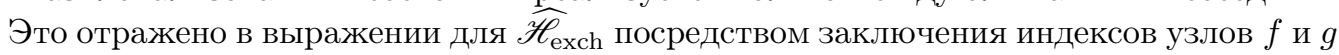
у знака суммы в угловые скобки. В двухподрешеточном описании параметр $W_{f g}$ обозначает интеграл гибридизации коллективизированных и квазилокализованных состояний, относящихся к разным подрешеткам. Для интенсивности гибридизационных процессов внутри одной подрешетки оставлены прежние обозначения $V_{f f^{\prime}}, V_{g g^{\prime}}$.

\section{3. ОПИСАНИЕ ФАЗЫ СОСУЩЕСТВОВАНИЯ СП И АФМ С ПОМОЩЬЮ БАЗИСНЫХ ОПЕРАТОРОВ}

Для получения замкнутой системы уравнений самосогласования, описывающей сверхпроводящую фазу, антиферромагнитную фазу, а также фазу сосуществования СП и АФМ, воспользуемся методом уравнений движения для двухвременных запаздывающих функций Грина [30], [31]. Замыкание цепочки уравнений движения будем осуществлять на основе метода неприводимых функций Грина [32], [33], применяя технику проецирования Цванцига-Мори [34], [35]. С этой целью введем базисный набор операторов

$$
\left\{X_{f}^{0 \sigma}, Y_{g}^{0 \sigma}, a_{f \sigma}, b_{g \sigma}, X_{f}^{\bar{\sigma} 0}, Y_{g}^{\bar{\sigma} 0}, a_{f \bar{\sigma}}^{\dagger}, b_{g \bar{\sigma}}^{\dagger}\right\}
$$

и составим точные уравнения движения для первой половины этого набора (уравнения движения для второй половины получаются из представленных ниже уравнений путем эрмитова сопряжения и замены $\sigma \rightarrow \bar{\sigma}=-\sigma)$ :

$$
\begin{aligned}
i \frac{d X_{f}^{0 \sigma}}{d t}= & \left(E_{0}-\mu\right) X_{f}^{0 \sigma}+\sum_{f^{\prime}} V_{f^{\prime} f}^{*}\left[\left(X_{f}^{00}+X_{f}^{\sigma \sigma}\right) a_{f^{\prime} \sigma}+X_{f}^{\bar{\sigma} \sigma} a_{f^{\prime} \bar{\sigma}}\right]+ \\
& +\sum_{g} W_{g f}^{*}\left[\left(X_{f}^{00}+X_{f}^{\sigma \sigma}\right) b_{g \sigma}+X_{f}^{\bar{\sigma} \sigma} b_{g \bar{\sigma}}\right]+\frac{1}{2} \sum_{g} J_{f g}\left(X_{f}^{0 \bar{\sigma}} Y_{g}^{\bar{\sigma} \sigma}-X_{f}^{0 \sigma} Y_{g}^{\bar{\sigma} \bar{\sigma}}\right),
\end{aligned}
$$




$$
\begin{aligned}
i \frac{d Y_{g}^{0 \sigma}}{d t}= & \left(E_{0}-\mu\right) Y_{g}^{0 \sigma}+\sum_{g^{\prime}} V_{g^{\prime} g}^{*}\left[\left(Y_{g}^{00}+Y_{g}^{\sigma \sigma}\right) b_{g^{\prime} \sigma}+Y_{g}^{\bar{\sigma} \sigma} b_{g^{\prime} \bar{\sigma}}\right]+ \\
& +\sum_{f} W_{f g}^{*}\left[\left(Y_{g}^{00}+Y_{g}^{\sigma \sigma}\right) a_{f \sigma}+Y_{g}^{\bar{\sigma} \sigma} a_{f \bar{\sigma}}\right]+\frac{1}{2} \sum_{f} J_{f g}\left(X_{f}^{\bar{\sigma} \sigma} Y_{g}^{0 \bar{\sigma}}-X_{f}^{\bar{\sigma} \bar{\sigma}} Y_{g}^{0 \sigma}\right), \\
i \frac{d a_{f \sigma}}{d t}= & \left(\varepsilon_{0}-\mu\right) a_{f \sigma}+\sum_{f^{\prime}}\left(t_{f f^{\prime}} a_{f^{\prime} \sigma}+V_{f f^{\prime}} X_{f^{\prime}}^{0 \sigma}\right)+\sum_{g}\left(t_{f g} b_{g \sigma}+W_{f g} Y_{g}^{0 \sigma}\right), \\
i \frac{d b_{g \sigma}}{d t}= & \left(\varepsilon_{0}-\mu\right) b_{g \sigma}+\sum_{g^{\prime}}\left(t_{g g^{\prime}} b_{g^{\prime} \sigma}+V_{g g^{\prime}} Y_{g^{\prime}}^{0 \sigma}\right)+\sum_{f}\left(t_{g f} a_{f \sigma}+W_{g f} X_{f}^{0 \sigma}\right),
\end{aligned}
$$

где индекс * означает комплексное сопряжение.

Проецирование правых частей этих уравнений на базис (1) и переход в квазиимпульсное представление позволяют получить замкнутую систему восьми уравнений для нормальных и аномальных функций Грина. В матричной форме эта система записывается следующим образом:

$$
\left(\begin{array}{cc}
\widehat{M}_{p \sigma}(\omega) & \widehat{G}_{p \sigma} \\
-\widehat{G}_{-p \bar{\sigma}}^{*} & -\widehat{M}_{-p \bar{\sigma}}^{*}(-\omega)
\end{array}\right)\left\langle\left\langle\vec{A} \mid B^{\dagger}\right\rangle\right\rangle_{\omega}=\left\langle\left\{\vec{A}, B^{\dagger}\right\}_{+}\right\rangle .
$$

Входящий в векторную функцию Грина оператор $\vec{A}$ определяется через фурье-образы базисных операторов (1):

$$
\vec{A}=\left(X_{p \sigma}, Y_{p \sigma}, a_{p \sigma}, b_{p \sigma}, X_{-p \bar{\sigma}}^{\dagger}, Y_{-p \bar{\sigma}}^{\dagger}, a_{-p \bar{\sigma}}^{\dagger}, b_{-p \bar{\sigma}}^{\dagger}\right) .
$$

Оператор $B$ выбирается произвольно из набора (3). Как обычно в методе двухвременных функций Грина, при учете только квазифермиевских возбуждений свободные члены каждого уравнения определяются термодинамическим средним $\langle\cdot\rangle$ от антикоммутатора $\{\cdot, \cdot\}_{+}$компоненты вектора $\vec{A}$ и оператора $B$. При записи уравнений (2) предполагалось, что $\left\langle A B^{\dagger}\right\rangle^{*}=\left\langle B A^{\dagger}\right\rangle$.

В дальнейшем существенную роль будут играть средние от антикоммутаторов операторов Хаббарда в квазиимпульсном представлении:

$$
\left\langle\left\{X_{p \sigma}, X_{p \sigma}^{\dagger}\right\}_{+}\right\rangle=\left\langle\left\{Y_{p \bar{\sigma}}, Y_{p \bar{\sigma}}^{\dagger}\right\}_{+}\right\rangle=\alpha_{\sigma}, \quad \alpha_{\sigma}=1-\frac{n_{\mathrm{L}}}{2}+\eta_{\sigma} R .
$$

Здесь величина намагниченности подрешетки $F$ обозначена через $R=\left\langle S_{f}^{z}\right\rangle$, а среднее число электронов на локализованном уровне $\left\langle N_{f}\right\rangle=\left\langle N_{g}\right\rangle=n_{\mathrm{L}}$. Зависящая от $\sigma$ функция $\eta_{\sigma}$ определяется обычным образом: $\eta_{\sigma}= \pm 1$, если $\sigma= \pm 1 / 2$ соответственно. Введенные в (2) матрицы четвертого порядка задаются выражениями

$$
\begin{aligned}
\widehat{M}_{p \sigma}(\omega) & =\left(\begin{array}{cccc}
\omega-E_{\sigma} & L_{p} / \alpha_{\bar{\sigma}} & -\alpha_{\sigma} V_{p}^{*} & -\alpha_{\sigma} W_{p}^{*} \\
L_{p} / \alpha_{\sigma} & \omega-E_{\bar{\sigma}} & -\alpha_{\bar{\sigma}} W_{p}^{*} & -\alpha_{\bar{\sigma}} V_{p}^{*} \\
-V_{p} & -W_{p} & \omega-\xi_{p} & -\Gamma_{p} \\
-W_{p} & -V_{p} & -\Gamma_{p} & \omega-\xi_{p}
\end{array}\right), \\
\widehat{G}_{p \sigma} & =-\left(\begin{array}{cccc}
\eta_{\sigma} \Phi / \alpha_{\bar{\sigma}} & \eta_{\sigma} \Delta_{p} / \alpha_{\sigma} & 0 & 0 \\
\eta_{\sigma} \Delta_{-p} / \alpha_{\bar{\sigma}} & \eta_{\sigma} \Phi / \alpha_{\sigma} & 0 & 0 \\
0 & 0 & 0 & 0 \\
0 & 0 & 0 & 0
\end{array}\right)
\end{aligned}
$$


В этих формулах ренормированное выражение для энергии локализованного уровня,

$$
E_{\sigma}=E_{0}-\mu-J\left(n_{\mathrm{L}}+2 \eta_{\sigma} R\right)-\Lambda_{\sigma}+C_{\sigma}
$$

описывает среднеполевое влияние обменного взаимодействия, приводящего как к смещению уровня (вклад, пропорциональный $-J n_{\mathrm{L}}$ ), так и к снятию вырождения этого уровня по проекции спинового момента (вклад, пропорциональный $-2 \eta_{\sigma} J R$ ). Третье слагаемое

$$
\Lambda_{\sigma}=\frac{1}{\alpha_{\sigma}} \cdot \frac{1}{N} \sum_{k}\left\{V_{k}^{*}\left\langle X_{k \bar{\sigma}}^{\dagger} a_{k \bar{\sigma}}\right\rangle+W_{k}^{*}\left\langle X_{k \bar{\sigma}}^{\dagger} b_{k \bar{\sigma}}\right\rangle\right\}
$$

возникает из-за совместного влияния кинематического и гибридизационного взаимодействий. Кинематическое взаимодействие является следствием нефермиевского характера перестановочных соотношений для операторов Хаббарда [36], [37], описывающих подсистему f-электронов. Последнее слагаемое в ренормированном выражении для энергии (4),

$$
C_{\sigma}=\frac{1}{\alpha_{\sigma}} \cdot \frac{1}{2 N} \sum_{k} J_{k} C_{k \sigma}
$$

связано с учетом статических магнитных флуктуаций и флуктуаций величины заполнения f-уровня. Эти флуктуации посредством механизма обменной связи приводят к сдвигу локализованного уровня, зависящему от проекции спинового момента. Входящая в выражение (5) величина $C_{k \sigma}$ является фурье-образом пространственного коррелятора

$$
C_{f g}^{\sigma}=\left\langle X_{f}^{\sigma \bar{\sigma}} Y_{g}^{\bar{\sigma} \sigma}\right\rangle+\frac{1}{4}\left\langle\widehat{N}_{f} \widehat{N}_{g}\right\rangle+\left\langle S_{f}^{z} S_{g}^{z}\right\rangle-\left(\frac{1}{4} n_{\mathrm{L}}^{2}-R^{2}\right)
$$

и задается равенством

$$
C_{f g}^{\sigma}=\frac{1}{N} \sum_{k} e^{i k(f-g)} C_{k \sigma}
$$

В формуле (6) мы учли, что $\left\langle X_{f}^{\uparrow \uparrow} Y_{g}^{\uparrow \uparrow}\right\rangle=\left\langle X_{f}^{\downarrow \downarrow} Y_{g}^{\downarrow \downarrow}\right\rangle$.

Совместное влияние кинематического и обменного взаимодействий в подсистеме локализованных фермионов приводит к возникновению эффективной связи между локализованными состояниями из разных подрешеток (в исходном гамильтониане соответствующие слагаемые отсутствовали). Интенсивность такого перемешивания определяется функцией $L_{p}$, которая выражается через кинетические корреляторы:

$$
L_{p}=\frac{1}{2 N} \sum_{k} J_{p-k}\left\langle Y_{k \uparrow}^{\dagger} X_{k \uparrow}\right\rangle
$$

Как известно, возникновение сверхпроводящего состояния на математическом языке описывается путем введения аномальных средних от фермиевских и квазифермиевских операторов. Присутствие в расширенной модели Андерсона гибридизационного, обменного и кинематического взаимодействий приводит к возможности 
реализации двух типов аномальных средних. Соответственно в теории возникают два сверхпроводящих параметра порядка (СПП). Первый из них,

$$
\Phi=\frac{1}{N} \sum_{k, \sigma} \eta_{\sigma}\left(V_{k}^{*}\left\langle a_{k \sigma} X_{-k \bar{\sigma}}\right\rangle+W_{k}^{*}\left\langle b_{k \sigma} X_{-k \bar{\sigma}}\right\rangle\right),
$$

связан с аномальными спариваниями коллективизированных и локализованных фермионов, которые индуцированы совместным влиянием гибридизационного и кинематического взаимодействий. Второй СПП является результатом куперовского спаривания квазилокализованных фермионов из разных подрешеток и обуславливается динамической частью обменного взаимодействия:

$$
\Delta_{p}=\frac{1}{2 N} \sum_{k} J_{p-k}\left(\left\langle X_{k \uparrow} Y_{-k \downarrow}\right\rangle+\left\langle Y_{-k \uparrow} X_{k \downarrow}\right\rangle\right) .
$$

Величина $\xi_{p}=\varepsilon_{0}+t_{p}-\mu$ соответствует отсчитанной от химического потенциала $\mu$ той части кинетической энергии коллективизированных электронов, которая связана с внутриподрешеточными перескоками. Функции $t_{p}, \Gamma_{p}, V_{p}$ и $W_{p}$ определяются через фурье-преобразования внутриподрешеточных и межподрешеточных параметров перескоков и гибридизации:

$$
\begin{aligned}
t_{f f^{\prime}} & =\frac{1}{N} \sum_{k} e^{i k\left(f-f^{\prime}\right)} t_{k}, & t_{f g} & =\frac{1}{N} \sum_{k} e^{i k(f-g)} \Gamma_{k}, \\
V_{f f^{\prime}} & =\frac{1}{N} \sum_{k} e^{i k\left(f-f^{\prime}\right)} V_{k}, & W_{f g} & =\frac{1}{N} \sum_{k} e^{i k(f-g)} W_{k} .
\end{aligned}
$$

При проецировании уравнений движения на базисный набор операторов (1) мы учли очевидную связь аномальных средних $\left\langle X_{f}^{0 \sigma} a_{f^{\prime} \bar{\sigma}}\right\rangle,\left\langle X_{f}^{0 \sigma} b_{g \bar{\sigma}}\right\rangle$ с аномальными средними $\left\langle Y_{g}^{0 \sigma} b_{g^{\prime} \bar{\sigma}}\right\rangle,\left\langle Y_{g}^{0 \sigma} a_{f \bar{\sigma}}\right\rangle$. Соответствующие формулы получаются на основе простых соображений симметрии. Применив операцию трансляции, переводящую узлы подрешетки $F$ в узлы подрешетки $G$, и инверсию оси квантования $z$, получим, что $\left\langle Y_{g}^{0 \sigma} b_{g^{\prime} \bar{\sigma}}\right\rangle=-\left\langle X_{f}^{0 \bar{\sigma}} a_{f^{\prime} \sigma}\right\rangle,\left\langle Y_{g}^{0 \sigma} a_{f \bar{\sigma}}\right\rangle=-\left\langle X_{f}^{0 \bar{\sigma}} b_{g \sigma}\right\rangle$. При этом был использован закон преобразования для спинорных величин при повороте вокруг оси $x$ :

$$
\begin{aligned}
c_{l \sigma} & \rightarrow c_{l \sigma}(\theta)=e^{i \theta s_{l}^{x}} c_{l \sigma} e^{-i \theta s_{l}^{x}}=c_{l \sigma} \cos \frac{\theta}{2}-i c_{l \bar{\sigma}} \sin \frac{\theta}{2} \\
X_{l}^{0 \sigma} & \rightarrow X_{l}^{0 \sigma}(\theta)=e^{i \theta S_{l}^{x}} X_{l}^{0 \sigma} e^{-i \theta S_{l}^{x}}=X_{l}^{0 \sigma} \cos \frac{\theta}{2}-i X_{l}^{0 \bar{\sigma}} \sin \frac{\theta}{2} .
\end{aligned}
$$

\section{4. НЕПРИВОДИМЫЕ ФУНКЦИИ ГРИНА} И УРАВНЕНИЯ САМОСОГЛАСОВАНИЯ

При исследовании сосуществования СП и АФМ важное значение имеет тип симметрии СПП. В настоящее время для систем с тяжелыми фермионами этот вопрос остается открытым. Фурье-образы обменного интеграла $J_{p \pm k}$, входящие в определение (7), могут приводить к квазиимпульсной зависимости СПП $\Delta_{p}$, соответствующей тому или иному типу симметрии СПП. Для магнитной зоны Бриллюэна, преобразованной посредством поворота на угол $\pi / 4$ относительно исходной зоны Бриллюэна, зависимость СПП, отвечающая $s$-типу симметрии, принимает вид 
$\Delta_{p}^{s}=2 \Delta_{0}^{s} \cos \left(p_{x} b / 2\right) \cos \left(p_{y} b / 2\right)$. Уменьшение зоны Бриллюэна связано с переходом к новой элементарной ячейке с параметром $b=\sqrt{2} a$ решетки с двумя антиферромагнитными подрешетками. В преобразованной системе координат зависимость СПП для $d$-типа симметрии представляется в виде $\Delta_{p}^{d}=2 \Delta_{0}^{d} \sin \left(p_{x} b / 2\right) \sin \left(p_{y} b / 2\right)$. Отметим, что для соединения $\mathrm{CeRhIn}_{5}$ более предпочтительным считается $d$-тип симметрии СПП [38], поэтому ниже ограничимся исследованием взаимного влияния СП и АФМ с этим типом симметрии параметра порядка.

Выбор данного типа симметрии СПП приводит к упрощению уравнений самосогласования, поскольку аномальный параметр порядка $\Phi$ дает вклад только для $s$-типа симметрии. Поэтому в дальнейшем считается, что $\Phi=0$.

При записи решения уравнений (2) для функции Грина в фазе сосуществования СП и АФМ воспользуемся обозначениями

$$
\begin{aligned}
d_{3 \sigma}(p, \omega)= & \left(\omega+E_{\sigma}\right)\left(\left(\omega+\xi_{p}\right)^{2}-\Gamma_{p}^{2}\right)-\alpha_{\sigma}\left(\omega+\xi_{p}\right)\left(V_{p}^{2}+W_{p}^{2}\right)+2 \alpha_{\sigma} \Gamma_{p} V_{p} W_{p}, \\
f_{3}(p, \omega)= & \frac{L_{p}}{\alpha_{\sigma} \alpha_{\bar{\sigma}}}\left(\left(\omega+\xi_{p}\right)^{2}-\Gamma_{p}^{2}\right)+2\left(\omega+\xi_{p}\right) V_{p} W_{p}-\Gamma_{p}\left(V_{p}^{2}+W_{p}^{2}\right), \\
d_{4}(p, \omega)= & \left(\omega+E_{\bar{\sigma}}\right) d_{3 \sigma}(p, \omega)+\left(\omega+E_{\sigma}\right) d_{3 \bar{\sigma}}(p, \omega)+\alpha_{\sigma} \alpha_{\bar{\sigma}}\left(V_{p}^{2}-W_{p}^{2}\right)^{2}- \\
& -\left(\left(\omega+E_{\sigma}\right)\left(\omega+E_{\bar{\sigma}}\right)-\frac{L_{p}^{2}}{\alpha_{\sigma} \alpha_{\bar{\sigma}}}\right)\left(\left(\omega+\xi_{p}\right)^{2}-\Gamma_{p}^{2}\right)-2 L_{p} f_{3}(p, \omega) .
\end{aligned}
$$

Уравнение $d_{4}(p,-\omega)=0$ определяет энергетический спектр системы в нормальной антиферромагнитной фазе, когда не учитываются аномальные средние.

Выражения для нормальных функций Грина записываются в виде

$$
\left\langle\left\langle X_{p \sigma} \mid X_{p \sigma}^{\dagger}\right\rangle_{\omega}=-\frac{\alpha_{\sigma} S_{p \sigma}(\omega)}{D_{8}(p, \omega)}, \quad\left\langle\left\langle a_{p \sigma} \mid a_{p \sigma}^{\dagger}\right\rangle_{\omega}=\frac{C_{p \sigma}(\omega)}{D_{8}(p, \omega)},\right.\right.
$$

где

$$
\begin{aligned}
S_{p \sigma}(\omega)= & d_{3 \bar{\sigma}}(p,-\omega) d_{4}(p, \omega)+\left(\frac{\Delta_{p}}{\alpha_{\bar{\sigma}}}\right)^{2}\left(\left(\omega-\xi_{p}\right)^{2}-\Gamma_{p}^{2}\right) d_{3 \sigma}(p, \omega), \\
C_{p \sigma}(\omega)= & \left(\left(\omega-E_{\sigma}\right)\left(\omega-E_{\bar{\sigma}}\right)-\frac{L_{p}^{2}}{\alpha_{\sigma} \alpha_{\bar{\sigma}}}\right)\left(\omega-\xi_{p}\right) d_{4}(p, \omega)- \\
& -\left(\alpha_{\bar{\sigma}} V_{p}^{2}\left(\omega-E_{\sigma}\right)+\alpha_{\sigma} W_{p}^{2}\left(\omega-E_{\bar{\sigma}}\right)-2 L_{p} V_{p} W_{p}\right) d_{4}(p, \omega)- \\
& -\left(\frac{\Delta_{p}}{\alpha_{\bar{\sigma}}}\right)^{2}\left(\left(\omega-E_{\sigma}\right)\left(\omega-\xi_{p}\right)-\alpha_{\sigma} W_{p}^{2}\right) d_{3 \sigma}(p, \omega)- \\
& -\left(\frac{\Delta_{p}}{\alpha_{\sigma}}\right)^{2}\left(\left(\omega-E_{\bar{\sigma}}\right)\left(\omega-\xi_{p}\right)-\alpha_{\bar{\sigma}} V_{p}^{2}\right) d_{3 \bar{\sigma}}(p, \omega)- \\
& -2 \Delta_{p}^{2}\left(V_{p} W_{p}-\frac{L_{p}}{\alpha_{\sigma} \alpha_{\bar{\sigma}}}\left(\omega-\xi_{p}\right)\right) f_{3}(p, \omega)+\frac{\Delta_{p}^{4}\left(\omega-\xi_{p}\right)}{\left(\alpha_{\sigma} \alpha_{\bar{\sigma}}\right)^{2}}\left(\left(\omega+\xi_{p}\right)^{2}-\Gamma_{p}^{2}\right) .
\end{aligned}
$$

В силу эквивалентности антиферромагнитных подрешеток имеют место равенства функций Грина $\left\langle\left\langle X_{p \sigma} \mid X_{p \sigma}^{\dagger}\right\rangle\right\rangle_{\omega}=\left\langle\left\langle Y_{p \bar{\sigma}} \mid Y_{p \bar{\sigma}}^{\dagger}\right\rangle_{\omega}\right.$ и $\left\langle\left\langle a_{p \sigma} \mid a_{p \sigma}^{\dagger}\right\rangle_{\omega}=\left\langle\left\langle b_{p \bar{\sigma}} \mid b_{p \bar{\sigma}}^{\dagger}\right\rangle\right\rangle_{\omega}\right.$.

При записи аномальных функций Грина введем дополнительные обозначения

$$
Q_{p \sigma}(\omega)=\frac{1}{\alpha_{\bar{\sigma}}} d_{3 \sigma}(p, \omega) d_{3 \sigma}(p,-\omega)+\alpha_{\bar{\sigma}} f_{3}(p, \omega) f_{3}(p,-\omega),
$$




$$
R_{p \sigma}(\omega)=\frac{1}{\alpha_{\sigma}^{2} \alpha_{\bar{\sigma}}}\left(\left(\omega+\xi_{p}\right)^{2}-\Gamma_{p}^{2}\right)\left(\left(\omega-\xi_{p}\right)^{2}-\Gamma_{p}^{2}\right)
$$

Тогда аномальная функция Грина записывается как

$$
\left\langle\left\langle Y_{-p \bar{\sigma}} \mid X_{p \sigma}\right\rangle_{\omega}=-\eta_{\bar{\sigma}} \alpha_{\sigma} \frac{\Delta_{p} Q_{p \bar{\sigma}}(\omega)+\Delta_{p}^{3} R_{p \bar{\sigma}}(\omega)}{D_{8}(p, \omega)} .\right.
$$

Отметим, что числитель выражения (12) содержит только четные степени по $\omega$.

Дисперсионное уравнение в фазе сосуществования СП и АФМ определяется условием наличия полюсов у функций Грина и имеет вид

$$
\begin{aligned}
0=D_{8}(p, \omega)= & d_{4}(p, \omega) d_{4}(p,-\omega)+\left(\frac{\Delta_{p}}{\alpha_{\bar{\sigma}}}\right)^{2} d_{3 \sigma}(p, \omega) d_{3 \sigma}(p,-\omega)+ \\
& +\left(\frac{\Delta_{p}}{\alpha_{\sigma}}\right)^{2} d_{3 \bar{\sigma}}(p, \omega) d_{3 \bar{\sigma}}(p,-\omega)+2 \Delta_{p}^{2} f_{3}(p, \omega) f_{3}(p,-\omega)+ \\
& +\left(\frac{\Delta_{p}^{2}}{\alpha_{\sigma} \alpha_{\bar{\sigma}}}\right)^{2}\left(\left(\omega-\xi_{p}\right)^{2}-\Gamma_{p}^{2}\right)\left(\left(\omega+\xi_{p}\right)^{2}-\Gamma_{p}^{2}\right) .
\end{aligned}
$$

Средние $\left\langle X_{f}^{\sigma \sigma}\right\rangle$, входящие в определение концентрации локализованных квазичастиц $n_{\mathrm{L}}$ и намагниченности $R$, можно связать с помощью спектральной теоремы с функциями Грина (11) (для различных направлений проекции спинового момента $\sigma)$. Поэтому такие средние определяются уравнениями самосогласования

$$
\left\langle X_{f}^{\sigma \sigma}\right\rangle=\frac{1}{N} \sum_{k, j} \alpha_{\sigma} \frac{S_{k \sigma}\left(-E_{j k}\right) f\left(-E_{j k} / T\right)-S_{k \sigma}\left(E_{j k}\right) f\left(E_{j k} / T\right)}{2 E_{j k} \prod_{i \neq j}\left(E_{j k}^{2}-E_{i k}^{2}\right)},
$$

где $f(x)=1 /\left(e^{x}+1\right)$ - функция распределения Ферми-Дирака, индексы $j, i$ пробегают значения от 1 до 4, что соответствует четырем положительно определенным ветвям энергетического спектра $E_{j k}$, найденным из дисперсионного уравнения (13). Таким образом величины $n_{\mathrm{L}}$ и $R$ определяются как

$$
n_{\mathrm{L}}=\left\langle X_{f}^{\uparrow \uparrow}\right\rangle+\left\langle X_{f}^{\downarrow \downarrow}\right\rangle, \quad R=\frac{\left\langle X_{f}^{\uparrow \uparrow}\right\rangle-\left\langle X_{f}^{\downarrow \downarrow}\right\rangle}{2} .
$$

Концентрация коллективизированных квазичастиц задается формулой

$$
n_{\mathrm{c}}=\frac{1}{N} \sum_{k, \sigma}\left\langle a_{k \sigma}^{\dagger} a_{k \sigma}\right\rangle
$$

где среднее выражается через определение функции Грина в (11):

$$
\left\langle a_{p \sigma}^{\dagger} a_{p \sigma}\right\rangle=\sum_{j} \frac{C_{p \sigma}\left(E_{j p}\right) f\left(E_{j p} / T\right)-C_{p \sigma}\left(-E_{j p}\right) f\left(-E_{j p} / T\right)}{2 E_{j p} \prod_{i \neq j}\left(E_{j p}^{2}-E_{i p}^{2}\right)} .
$$

Записав выражение для полного числа электронов в системе,

$$
N_{\mathrm{e}}=\sum_{f, \sigma}\left(\left\langle X_{f}^{\sigma \sigma}\right\rangle+\left\langle a_{f \sigma}^{\dagger} a_{f \sigma}\right\rangle\right)+\sum_{g, \sigma}\left(\left\langle Y_{g}^{\sigma \sigma}\right\rangle+\left\langle b_{g \sigma}^{\dagger} b_{g \sigma}\right\rangle\right),
$$


получим, что уравнение самосогласования для нахождения химического потенциала можно выразить через узельную концентрацию локализованных и коллективизированных электронов: $n_{\mathrm{e}}=N_{\mathrm{e}} / 2 N=n_{\mathrm{L}}+n_{\mathrm{c}}$.

Подставим $\Delta_{p}=2 \Delta_{0}^{d} \sin \left(p_{x} b / 2\right) \sin \left(p_{y} b / 2\right)$ в интегральное уравнение самосогласования, полученное из определения (7), с помощью спектральной теоремы для аномальных функций Грина (12) получим алгебраическое уравнение для амплитуды аномальных спариваний $\Delta_{0}^{d}$ :

$$
\begin{aligned}
1= & 2 J \frac{1}{N} \sum_{k}\left[\sin \left(\frac{k_{x} b}{2}\right) \sin \left(\frac{k_{y} b}{2}\right)\right]^{2} \sum_{j} \frac{-Q_{k}\left(E_{j k}\right) \operatorname{th}\left(E_{j k} / 2 T\right)}{2 E_{j k} \prod_{i \neq j}\left(E_{j k}^{2}-E_{i k}^{2}\right)}+ \\
& +8 J\left(\Delta_{0}^{d}\right)^{2} \frac{1}{N} \sum_{k}\left[\sin \left(\frac{k_{x} b}{2}\right) \sin \left(\frac{k_{y} b}{2}\right)\right]^{4} \sum_{j} \frac{-R_{k}\left(E_{j k}\right) \operatorname{th}\left(E_{j k} / 2 T\right)}{2 E_{j k} \prod_{i \neq j}\left(E_{j k}^{2}-E_{i k}^{2}\right)}
\end{aligned}
$$

где

$$
Q_{p}(\omega)=\sum_{\sigma} \alpha_{\sigma} Q_{p \bar{\sigma}}(\omega), \quad R_{p}(\omega)=\sum_{\sigma} \alpha_{\sigma} R_{p \bar{\sigma}}(\omega)
$$

\section{5. КВАЗИЧАСТИЧНЫЕ ЗОНЫ В ФАЗЕ СОСУЩЕСТВОВАНИЯ СП И АФМ}

Определитель $d_{4}(p, \omega)$ не зависит от направления проекции спинового момента электрона $\sigma$. Это свидетельствует об эквивалентности спектров фермиевских возбуждений в антиферромагнитной фазе для квазичастиц с направлением спинового момента $\sigma=\uparrow$ и $\sigma=\downarrow$. Нетрудно видеть, что определитель $D_{8}(p, \omega)$ также не зависит от направления проекции спинового момента.

Если в полном дисперсионном уравнении (13) положить $\Delta_{p}=0$, то возникающее при этом дисперсионное уравнение соответствует случаю нормальной фазы (уравнение $\left.d_{4}(p,-\omega)=0\right)$. В сверхпроводящей фазе дисперсионное уравнение обладает четырьмя дополнительными решениями. Их возникновение объясняется на рис. 1, где показан спектр квазичастичных зон в ПМА по главному направлению зоны Бриллюэна для концентраций $n_{\mathrm{e}}=1.4(\mathrm{a}, \mathrm{в})$ и $n_{\mathrm{e}}=1.2(б$, г). Энергетические параметры модели $J=0.2, V_{0}=0.6, E_{0}=-1.5$ нормированы на величину $\left|t_{1}\right|$. Параметры $\Lambda_{\sigma}, C_{\sigma}, L_{p}$ считаются равными нулю. Для простоты сравнения выбраны такие параметры модели, при которых система находится в нормальной фазе.

Решения уравнения $d_{4}(p,-\omega)=0$ при $n_{\mathrm{e}}=1.4$ для различных направлений проекций квазичастичного спинового момента представлены на рис. 1а. При такой концентрации самосогласованный расчет приводит к только тривиальному решению для намагниченности $R=0$ и значениям $n_{\mathrm{L}}=0.907, \mu=-1.554$. Штрихпунктирной линией на рис. 1 показан уровень химического потенциала $\mu$. Изменение концентрации может индуцировать переход в антиферромагнитную фазу. Например, при концентрации $n_{\mathrm{e}}=1.2$ в системе реализуется дальний антиферромагнитный порядок (см. рис. 1б) с параметрами $R=0.364, n_{\mathrm{L}}=0.746, \mu=-1.706$. Нижние две стрелки на правой и левой частях рис. $1 б$ указывают на то, что квазичастичные зоны раздвигаются; это вызвано обменным полем спиновых моментов локализованных электронов. Раздвижка ветвей, соответствующих состояниям зонных электронов (верхняя пара стрелок), обусловлена гибридизационными процессами с антиферромагнитно упорядоченными локализованными электронами. Вид квазичастичных 


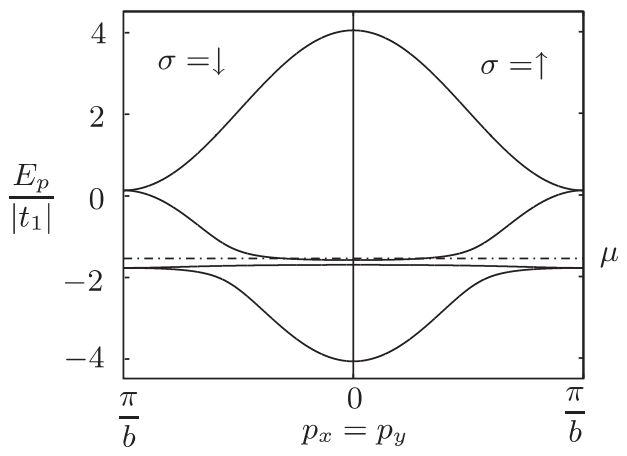

a

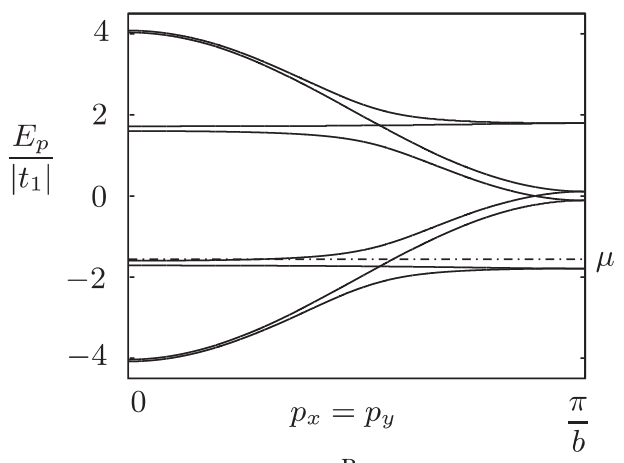

$\mathrm{B}$

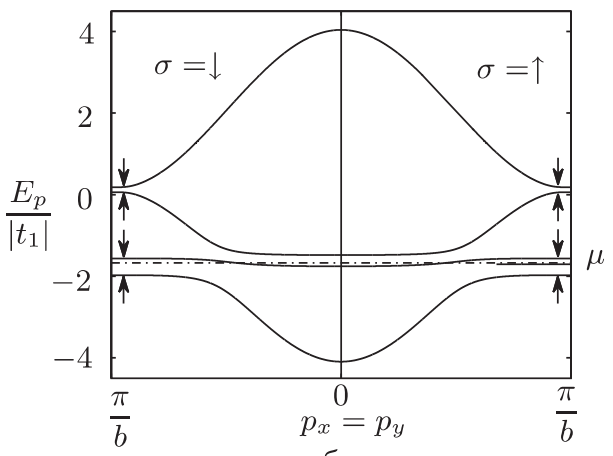

6

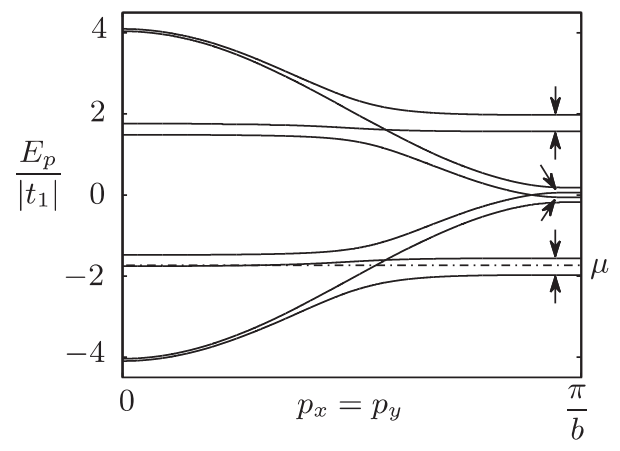

$\Gamma$

Рис. 1. Квазичастичный спектр ПМА, найденный из уравнения $d_{4}(p,-\omega)=$ 0 (см. (10) и графики а, б) и из уравнения (13) (графики в, г) для $J=0.2$, $V_{0}=0.6, E_{0}=-1.5$. На графиках приведены спектры для нормальной парамагнитной фазы $\left(\Delta_{0}^{d}=0, R=0\right)$ при $n_{\mathrm{e}}=1.4, \mu=-1.554, n_{\mathrm{L}}=0.907(\mathrm{a}, \mathrm{в})$ и нормальной антиферромагнитной фазы $\left(\Delta_{0}^{d}=0, R=0.364\right)$ при $n_{\mathrm{e}}=1.2$, $\mu=-1.706, n_{\mathrm{L}}=0.746(б$, г).

зон, представленный на рис. 1а, 1б, характерен для двухподрешеточной ПМА (см., например, статью [39]).

Решения уравнения (13) для тех же параметров, что и на рис. 1a, 1б, показаны на рис. 1в, 1г соответственно. Появление дополнительных ветвей связано с тем, что аномальные функции Грина построены на операторах с различным направлением квазичастичного спинового момента. В этом случае общий энергетический спектр в задаче о сосуществовании СП и АФМ включает в себя четыре энергетические ветви тяжелофермионного антиферромагнетика для квазичастиц с $\sigma=\uparrow$ и четыре ветви в случае $\sigma=\downarrow$, взятые с обратным знаком.

В общем случае аналитический вид решений дисперсионного уравнения (13) является достаточно громоздким и здесь не приводится. В практическом отношении важно иметь хоть и приближенные, но аналитические выражения для квазичастичного спектра, лежащего в окрестности энергии локализованных состояний $E_{0}$. Именно через них определяется дисперсионная зависимость зоны тяжелых фермионов. 
Получение приближенного выражения связано с заменой $\omega-\xi_{p} \rightarrow E_{J}-\mu-\xi_{p}$ в уравнении (13). Здесь использовано обозначение $E_{J}=E_{0}-J n_{\mathrm{L}}$. Описанное приближение справедливо при малом расщеплении зоны тяжелых фермионов в антиферромагнитной фазе, когда $4 J R \ll W[40]$, где $W$ - ширина зоны коллективизированных электронов. Для простоты мы ограничимся рассмотрением только внутриподрешеточной гибридизации $\left(W_{p}=0\right)$, а также приближением ближайших соседей относительно перескоков электронов. В дальнейшем отсчет энергии ведется от уровня $\varepsilon_{0}$, т. е. считается, что $\varepsilon_{0}=0$. В этом случае

$$
\begin{aligned}
{\left[E_{p}^{\mathrm{HF}}\right]^{2}=} & \left(\left(1-\alpha \gamma_{p}\right) E_{J}\right)^{2}+\left(\alpha^{2}-R^{2}\right) \Gamma_{p}^{2} \gamma_{p}^{2}+\left(\gamma_{p} E_{J}+2 J\right)^{2} R^{2}+ \\
& +\frac{\alpha^{2}+R^{2}}{\left(\alpha^{2}-R^{2}\right)^{2}} \Delta_{p}^{2}+2 \operatorname{sgn}\left(\gamma_{p}\right) \nu_{p}^{2}, \quad \gamma_{p}=\frac{V_{p}^{2}}{\Gamma_{p}^{2}-E_{J}^{2}} .
\end{aligned}
$$

Индекс НF отражает тот факт, что выражение (15) описывает только спектр зоны тяжелых фермионов. Функция $\operatorname{sgn}(\cdot)$ определяется стандартно: $\operatorname{sgn}\left(\gamma_{p}\right)= \pm 1$, если $\gamma_{p} \gtrless 0$. Также введено обозначение

$$
\begin{aligned}
\nu_{p}^{4}= & \left(\left(\alpha^{2}-R^{2}\right)\left(\left(1-\alpha \gamma_{p}\right) E_{J}\right)^{2}+\frac{\Delta_{p}^{2} R^{2}}{\alpha^{2}-R^{2}}\right) \Gamma_{p}^{2} \gamma_{p}^{2}+ \\
& +\left(\left(\left(1-\alpha \gamma_{p}\right) E_{J}\right)\left(\gamma_{p} E_{J}+2 J\right)+\frac{\alpha \Delta_{p}^{2}}{\left(\alpha^{2}-R^{2}\right)^{2}}\right)^{2} R^{2}
\end{aligned}
$$

Область, включающая зону тяжелых фермионов на рис. 1а, 1б, в увеличенном масштабе представлена на рис. 2а, 26 соответственно. На левой половине каждого из графиков на рис. 2 сплошными линиями показаны точные решения уравнения (13). На правой половине штриховая линия, отмеченная как $E_{p}^{\mathrm{HF}}$, отвечает решению, рассчитанному по формуле (15). Аналитическое выражение для спектра квазичастичных зон, выделенных цифрами 1,2 , можно получить из соотношения (15), если поменять знак перед слагаемым $2 \operatorname{sgn}\left(\gamma_{p}\right) \nu_{p}^{2}$. Видно, что приближенное аналитическое решение хорошо согласуется с точным, рассчитанным численно. Штриховая линия, обозначенная символом $\star$, не соответствует реальной зоне и приведена для наглядности.

Спектр квазичастичных зон при концентрации $n_{\mathrm{e}}=1.35$, отвечающей фазе сосуществования СП и АФМ, представлен на рис. 2в. Параметры такой фазы суть $R=0.411, \Delta_{0}^{d}=0.00514, n_{\mathrm{L}}=0.871, \mu=-1.595$. Образование сверхпроводящей щели в спектре элементарных возбуждений при таких параметрах показано на рис. 2г. Здесь энергия $\mathcal{E}_{p}^{\mathrm{HF}}$ отсчитывается от энергии Ферми, $p_{\mathrm{F}}$ - импульс Ферми при нулевом СПП. Значение $\Delta_{p_{\mathrm{F}}}$, которому на графике отвечает пунктирная линия, определяет амплитуду аномальных спариваний на поверхности Ферми. Видно, что реальный размер сверхпроводящей щели в спектре существенно превышает значение $\Delta_{p_{\mathrm{F}}}$. Это связано с тем, что спектр тяжелых фермионов имеет более сложный вид по сравнению с энергетическим спектром в теории Бардина-Купера-Шриффера. Поэтому СПП определяется не только амплитудой сверхпроводящих спариваний $\Delta_{p}$, но и зависит от других параметров модели. Чтобы найти величину СПП, которая более точно чем $\Delta_{p_{F}}$ соответствует размеру сверхпроводящей щели, приведем приближенное выражение для энергетического спектра тяжелых фермионов:

$$
\mathcal{E}_{p}^{\mathrm{HF}} \approx \sqrt{\left(\mathcal{E}_{p}^{\mathrm{AFM}}\right)^{2}+\zeta_{p} \Delta_{p}^{2}}
$$




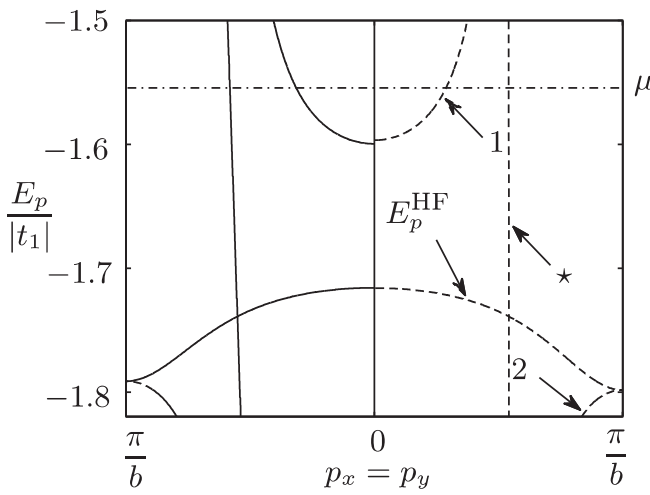

a

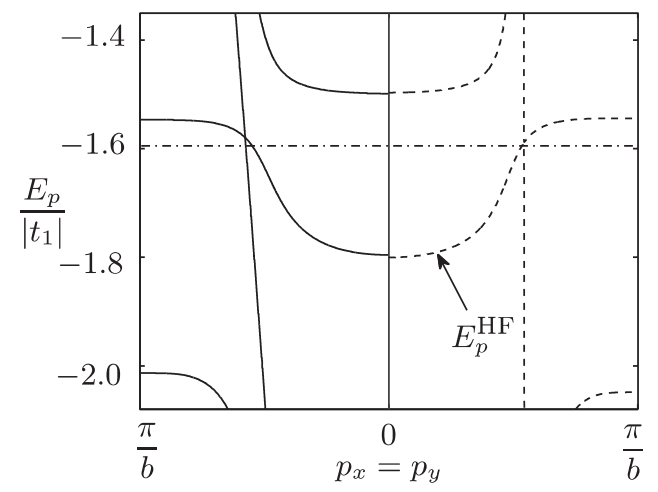

B

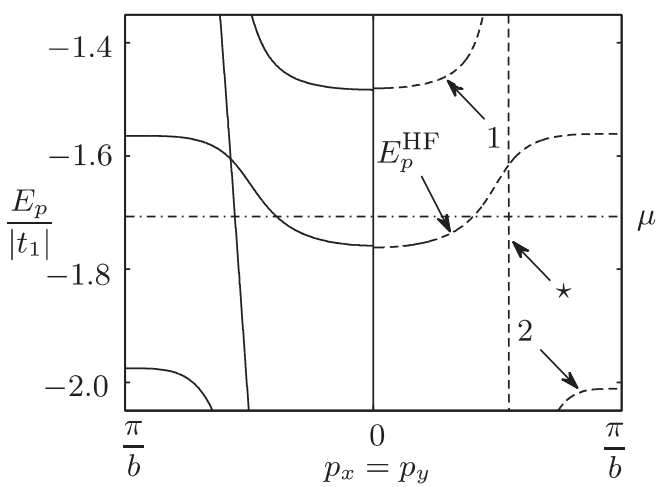

6

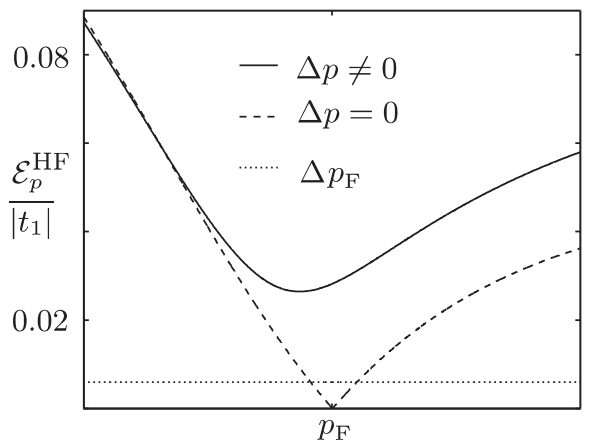

$\Gamma$

Рис. 2. Спектр зоны тяжелых фермионов $E_{p}^{\mathrm{HF}}$ ПМА: нормальная парамагнитная фаза $n_{\mathrm{e}}=1.4$ (а), нормальная антиферромагнитная фаза $n_{\mathrm{e}}=1.2$ (б); параметры на верхних графиках те же, что и на рис. 1a, 16 соответственно. Также приведены фаза сосуществования СП и АФМ $n_{\mathrm{e}}=1.35, R=0.411$, $\Delta_{0}^{d}=0.00514, n_{\mathrm{L}}=0.871, \mu=-1.595$ (в) и спектр элементарных возбуждений в фазе сосуществования (г).

где

$$
\begin{aligned}
\zeta_{p}= & \frac{\alpha^{2}+R^{2}}{\left(\alpha^{2}-R^{2}\right)^{2}}-\frac{R^{2}}{\left(\alpha^{2}-R^{2}\right)\left|\left(1-\alpha \gamma_{p}\right) E_{J}-\mu\right| \lambda_{p}} \times \\
& \times\left(\Gamma_{p}^{2} \gamma_{p}^{2}+\frac{2 \alpha}{\alpha^{2}-R^{2}}\left(\left(1-\alpha \gamma_{p}\right) E_{J}-\mu\right)\left(\gamma_{p} E_{J}+2 J\right)\right), \\
\mathcal{E}_{p}^{\mathrm{AFM}}= & \left|\left(1-\alpha \gamma_{p}\right) E_{J}-\mu\right|-\lambda_{p}, \quad \lambda_{p}=\sqrt{\left(\alpha^{2}-R^{2}\right) \Gamma_{p}^{2} \gamma_{p}^{2}+\left(\gamma_{p} E_{J}+2 J\right)^{2} R^{2}} .
\end{aligned}
$$

Нетрудно показать, что истинный СПП имеет вид $\Psi_{p}=\sqrt{\zeta_{p}} \Delta_{p}$. Однако переход из сверхпроводящей в нормальную фазу по-прежнему определяется условием $\Delta_{p}=0$. В этом случае величина $\Delta_{p}$ может определяться как СПП, но она не соответствует реальному размеру щели в спектре. 


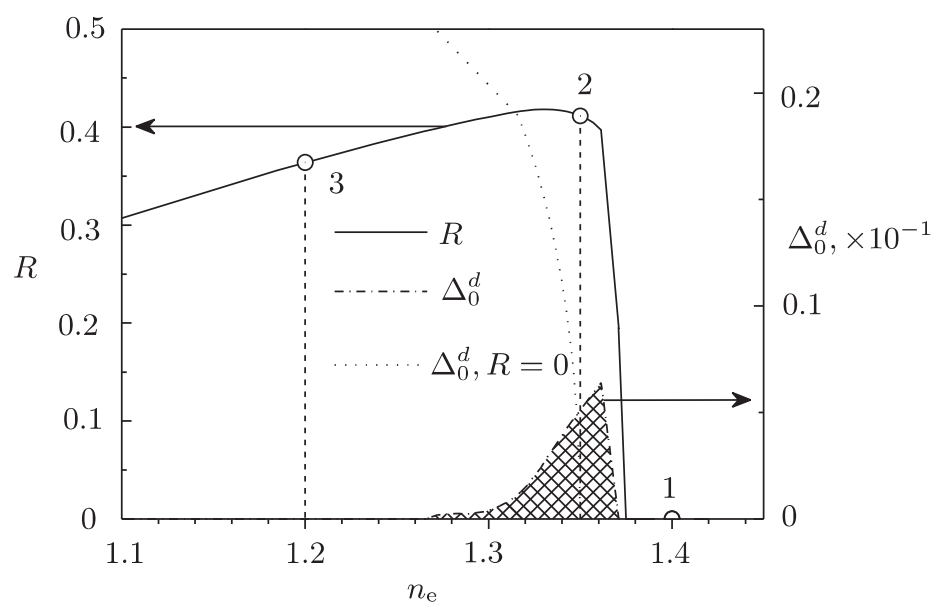

Рис. 3. Зависимость намагниченности подрешетки $R$ и амплитуды сверхпроводящего параметра порядка $\Delta_{0}^{d}$ от полной концентрации $n_{\mathrm{e}}$ носителей в системе. Параметры модели выбраны как $J=0.2, V_{0}=0.6, E_{0}=-1.5$.

Как отмечалось выше, приближенное выражение (15) отвечает спектру зоны тяжелых фермионов, эффективная масса которых превышает массу блоховских электронов в решетке. Существенно, что для реализации дальнего антиферромагнитного порядка и СП химический потенциал должен лежать в слабодисперсной зоне тяжелых фермионов. Поэтому антиферромагнитные и сверхпроводящие характеристики системы определяются именно тяжелыми фермионами. Для оценки эффективной массы новых квазичастиц разложим спектр (15) в окрестности точки $\left(p_{x}, p_{y}\right)=(0,0)$. Тогда масса тяжелых фермионов, энергетические зоны $E_{p}^{\mathrm{HF}}$ которых представлены на рис. 2а-2в, задается вблизи дна зоны следующим выражением:

$$
\frac{m_{\mathrm{HF}}}{m_{0}}=\frac{\Gamma_{0}^{2}-E_{J}^{2}}{\left|\Gamma_{0}\right| \gamma_{0}}\left(2 \alpha\left|E_{J}\right|-\frac{\left(\alpha^{2}-R^{2}\right) \Gamma_{0}^{2} \gamma_{0}+\left(\alpha^{2}+R^{2}\right) E_{J}^{2} \gamma_{0}-J_{0} R^{2}\left|E_{J}\right|}{\lambda_{0}}\right)^{-1},
$$

где $m_{0}=\hbar^{2} /\left|t_{1}\right| b^{2}$ - масса блоховского электрона на квадратной решетке. Величины $\Gamma_{0}, \gamma_{0}$ получаются из известных $\Gamma_{p}, \gamma_{p}$ при $p_{x}=0, p_{y}=0$. Подобная методика, но в рамках слейв-бозонного представления была применена в работе [40] для оценки массы тяжелых фермионов антиферромагнитных интерметаллидов при учете скоса антиферромагнитных подрешеток.

\section{6. СОСУЩЕСТВОВАНИЕ СП И АФМ В ТФ-СИСТЕМАХ}

Изменение типа основного состояния ПМА (при нулевой температуре) в зависимости от полной концентрации $n_{\mathrm{e}}$ носителей в системе представлено на рис. 3 при фиксированных параметрах $J=0.2, V_{0}=0.6, E_{0}=-1.5$. Сплошной линией показана намагниченность подрешетки $R$ (левая шкала), штрихпунктирной - амплитуда $\Delta_{0}^{d}$ аномальных спариваний, соответствующих $d$-типу симметрии (правая шкала). Результаты расчета $\Delta_{0}^{d}$ при нулевой намагниченности приведены для сравнения и изображены пунктирной линией. Цифрами 1, 2, 3 на графике обозначены 
состояния, энергетическая структура которых представлена на рис. 2а, 2б, 2в соответственно. Видно, что для зависимости намагниченности и амплитуды СПП от концентрации существенным является положение химического потенциала относительно зоны тяжелых фермионов.

Качественно картина заключается в следующем. При увеличении числа электронов за счет заполнения локализованного уровня в системе индуцируется дальний антиферромагнитный порядок. Однако последующее увеличение концентрации приводит к резкому подавлению АФМ. Это связано с тем, что уровень становится почти заполненным и добавляемые новые электроны формируют коллективизированную подсистему. Усиление гибридизационных процессов в таком случае является фактором, который отвечает за разрушение АФМ. Как показано на рис. 3, вблизи перехода из антиферромагнитной фазы в парамагнитную в системе реализуется куперовская неустойчивость. Это приводит к возникновению фазы сосуществования СП и АФМ. Заштрихованная область на рис. 3 определяет значения концентраций, при которых куперовская неустойчивость и дальний антиферромагнитный порядок сосуществуют.

Отметим, что в настоящем рассмотрении фазы ограничены не величиной температуры, а значениями концентраций. Наличие дальнего антиферромагнитного порядка в подсистеме локализованных хаббардовских фермионов может индуцировать сверхпроводящие спаривания. Это следует из того, что увеличение или уменьшение намагниченности приводит к аналогичному поведению амплитуды $\Delta_{0}^{d}$. K тому же куперовская неустойчивость становится характерной для значений концентрации, при которых она не проявляется, если не учитывать существования в системе антиферромагнитного упорядочения. С другой стороны, интенсивность аномальных спариваний при наличии АФМ существенно уменьшается.

Увеличение энергии локализованных состояний $E_{0}$ при фиксированной концентрации $n_{\mathrm{e}}=1.2$ и тех же параметрах $J$ и $V_{0}$, что и на рис. 3 , также приводит к разрушению антиферромагнитного упорядочения (см. рис. 4а). В данном случае это связано с тем, что, по мере того как локализованный уровень движется вверх по энергетической зоне, уменьшается заселенность локализованных состояний. При выбранных параметрах ПМА сверхпроводящее и антиферромагнитное упорядочения являются конкурирующими явлениями. На рис. 4а этот факт находит свое отражение в том, что максимум СПП достигается в точке падения намагниченности до нуля.

Хотя фаза сосуществования СП и АФМ реализуется, амплитуда СПП существенно ниже при конкуренции с дальним антиферромагнитным порядком. Описанная зависимость параметров порядка от энергии $E_{0}$ связана с экспериментальными исследованиями систем с тяжелыми фермионами. Например, упомянутое выше соединение $\mathrm{CeRhIn}_{5}$ при атмосферном давлении является антиферромагнетиком с температурой Нееля $T_{\mathrm{N}}=3.8 \mathrm{~K}$ [41]. Под действием давления температура Нееля образца сначала слабо уменьшается, а затем, при критическом давлении $P_{\mathrm{c}}=1.75$ ГПа, дальний антиферромагнитный порядок полностью разрушается [15]. Предположительно это происходит путем фазового перехода первого рода, который при нулевой температуре является квантовым фазовым переходом. В области давлений, меньших чем критическое $P_{\mathrm{c}}$, реализуется фаза сосуществования СП и АФМ [16]. При давлениях выше критического в экспериментах обнаружена чистая сверхпроводящая фаза. 


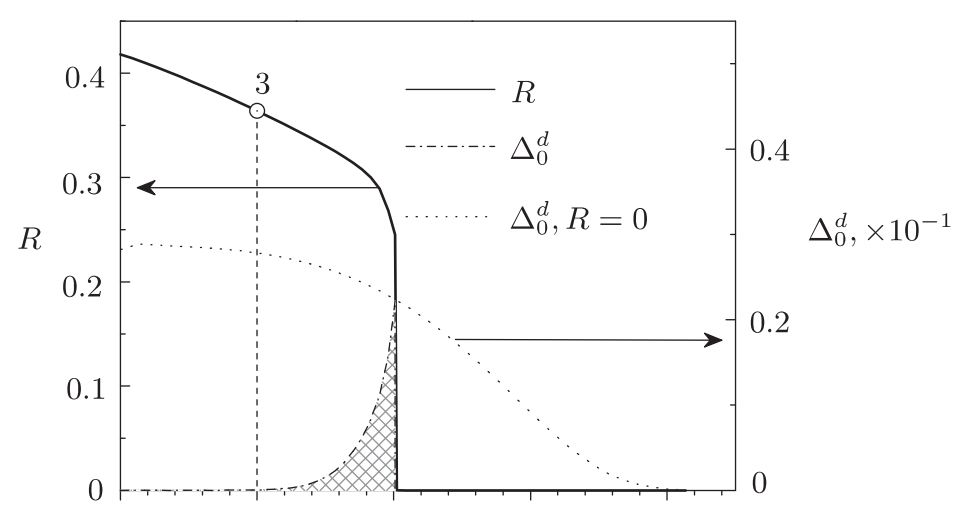

a

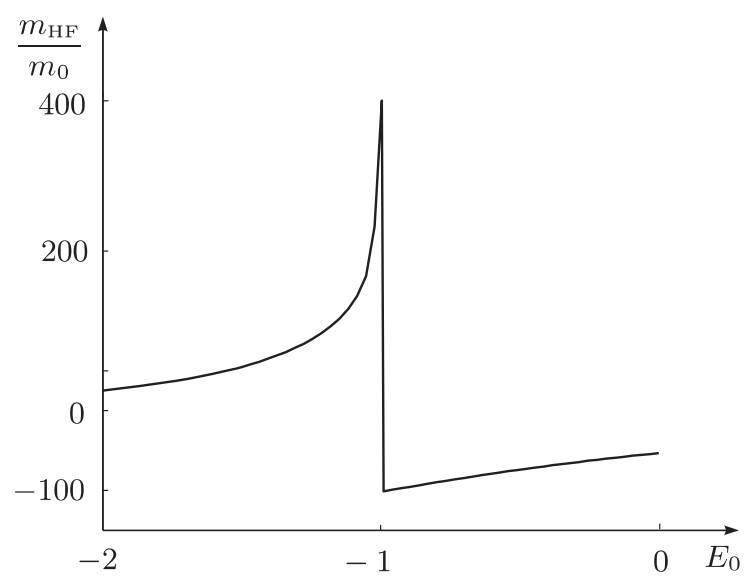

б

Рис. 4. Зависимость намагниченности подрешетки $R$ и амплитуды сверхпроводящего параметра порядка $\Delta_{0}^{d}$ от энергии локализованных состояний $E_{0}($ a). Зависимость эффективной массы тяжелых квазичастиц, нормированной на массу блоховского электрона, от энергии $E_{0}$ (б).

Описанным исследованиям качественно соответствует картина, представленная на рис. 4а. При этом роль основного параметра, который изменяется под воздействием внешнего гидростатического давления, играет энергия локализованных электронов $E_{0}$. Механизм увеличения энергии $E_{0}$ при росте давления достаточно прост. Известно, что церий входит в состав соединения $\mathrm{CeRhIn}_{5}$ как ион $\mathrm{Ce}^{3+}$, поэтому область вокруг иона обладает большим суммарным положительным зарядом. В силу условия электронейтральности ближайшее окружение обладает эффективно отрицательным зарядом. Сближение под действием давления иона церия с ближайшим окружением вызывает возрастание энергии 4f-электрона, находящегося на нем, за счет кулоновского взаимодействия. Отметим также, что возрастание давления может приводить, вообще говоря, к изменениям амплитуды перескока электронов между узлами $t_{m l}$ и параметра гибридизации $V_{m l}[22]$. В нашем рассмотрении пред- 
полагается, что изменение этих параметров при изменении давления вносит меньший вклад в формирование определенного типа основного состояния.

Важной характеристикой ТФ-систем является эффективная масса локализованных квазичастиц. Отношение эффективной массы локализованных квазичастиц в ПМА к массе коллективизированных электронов в приближении параболической зоны можно оценить по формуле (16). Зависимость этой величины от энергии локализованного уровня представлена на рис. 4б. При разрушении антиферромагнитного порядка масса (16) становится отрицательной. В таком случае говорят о переходе к дырочному типу тяжелых квазичастиц [40]. В каждой точке графика значения параметров порядка пересчитывались в соответствии с диаграммой основного состояния, представленной на рис. 4а. Видно, что по мере разрушения антиферромагнитного порядка, при увеличении $E_{0}$, эффективная масса растет. При этом в критической точке $\left(E_{0} \approx-1\right)$, выше которой намагниченность подрешетки равна нулю, эффективная масса демонстрирует поведение, похожее на расходимость. При переходе через критическую точку в парамагнитную область значение эффективной массы резко падает и в дальнейшем уменьшается по модулю.

На основе серии экспериментов, проведенных для соединения $\mathrm{CeRhIn}_{5}$, было показано увеличение массы электронов под действием внешнего давления [42], [43]. Так, при атмосферном давлении было зафиксировано, что циклотронная масса электронов превышает в 5.5 раза массу свободного электрона [42]. При критическом давлении это отношение могло достигать значения $m_{\mathrm{c}} / m_{0}=100$. Отметим, что в приближении параболической зоны эффективная и циклотронная массы электронов эквивалентны. Значения эффективной массы на рис. 4б, хоть и превышают значения циклотронной массы, качественно правильно отражают экспериментальную зависимость массы от давления. Отметим, что увеличение эффективной массы фермионов в описываемых системах связано с переходом из антиферромагнитной фазы в парамагнитную и с уменьшением ширины зоны тяжелых фермионов.

\section{7. ЗАКЛЮЧЕНИЕ}

Проведенное исследование показало, что наличие обменной связи в подсистеме локализованных фермионов и гибридизационного взаимодействия между локализованными и коллективизированными электронами является достаточным для реализации антиферромагнитной фазы, сверхпроводящей фазы, а также фазы сосуществования СП и АФМ. Роль контрольного параметра, определяющего фазовый переход в упорядоченное состояние при заданной суммарной концентрации электронов, играет положение затравочного энергетического уровня локализованных электронов относительно дна зоны проводимости. Предположение об изменении этого параметра при возрастании внешнего давления лежит в основе интерпретации экспериментально наблюдаемой модификации структуры основного состояния тяжелофермионного соединения $\mathrm{CeRhIn}_{5}$ в результате квантового фазового перехода.

На диаграмме состояний системы имеется значение контрольного параметра, в окрестности которого реализуется фаза сосуществования СП и АФМ. Приближение к критическому значению контрольного параметра со стороны низких температур сопровождается быстрым разрушением дальнего антиферромагнитного упорядочения с одновременным возрастанием СПП. Наличие дальнего антиферромагнитного 
упорядочения проявляется как в сильном подавлении амплитуды аномальных куперовских средних, так и в модификации фермиевского энергетического спектра. В результате величина сверхпроводящей щели определяется не только амплитудой аномальных средних, но и значением намагниченности антиферромагнитной подрешетки. Это означает, что ренормировка сверхпроводящей щели в фазе сосуществования СП и АФМ идет по двум каналам: первый из них связан с дальним магнитным порядком, а второй - с модификацией спектра фермиевских возбуждений.

Благодарности. Работа выполнена при поддержке Программы Президиума РАН "Квантовые мезоскопические и неупорядоченные структуры", РФФИ (гранты № 1002-00251, 11-02-98007-РФФИ-Сибирь) и Министерства образования и науки РФ (соглашение 14.132.21.1410).

\section{Список литературы}

[1] P. W. Anderson, Science, 235:4793 (1987), 1196-1198.

[2] N. M. Plakida, High-Temperature Cuprate Superconductors. Experiment, Theory, and Applications, Springer Series in Solid-State Sciences, 166, Springer, Heidelberg, Berlin, 2010.

[3] Н. Е. Алексеевский, Д. И. Хомский, УФН, 147:4 (1985), 767-779.

[4] G. R. Stewart, Rev. Mod. Phys., 56:4 (1987), 755-787.

[5] D. M. Newns, H. Read, Adv. Phys., 36:6 (1987), 799-849.

[6] В. В. Вальков, С. Г. Овчинников, Квазичастицы в сильно коррелированных системах, Изд-во СО РАН, Новосибирск, 2001.

[7] K. Miyake, S. Schmitt-Rink, C. M. Varma, Phys. Rev. B, 34:9 (1986), 6554-6556.

[8] Р. О. Зайцев, В. А. Иванов, ФТT, 29 (1987), 2554-2556.

[9] Ю. А. Изюмов, УФН, 161:11 (1991), 1-46.

[10] С. В. Вонсовский, М. С. Свирский, ЖЭТФ, 46:5 (1964), 1619-1631.

[11] А. И. Буздин, Л.Н.Булаевский, УФН, 149:1 (1986), 45-67.

[12] S. M. Hayden, L. Taillefer, C. Vettier, J. Flouquet, Phys. Rev. B, 46:13 (1992), 8675-8678.

[13] R. Caspary, P. Hellmann, M. Keller, G. Sparn, C. Wassiew, R. Köhler, C. Geibel, C. Schank, F. Steglich, N. E. Phillips, Phys. Rev. Lett., 71:13 (1993), 2146-2149.

[14] G. Zwicknagl, A. N. Yaresko, P. Fulde, Phys. Rev. B, 65:8 (2002), 081103, 4 pp.

[15] T. Park, J. D. Thompson, New J. Phys., 11 (2009), 055062, 17 pp., arXiv: 0908.2404.

[16] T. Mito, S. Kawasaki, Y. Kawasaki, G.-Q. Zheng, Y. Kitaoka, D. Aoki, Y. Haga, Y. Ōnuki, Phys. Rev. Lett., 90:7 (2003), 077004, 4 pp., arXiv: cond-mat/0211576.

[17] Ю. А. Изюмов, Н. М. Плакида, Ю. Н. Скрябин, УФН, 159:4 (1989), 621-663.

[18] H. Mukuda, Y. Yamaguchi, S. Shimizu, Y. Kitaoka, P. Shirage, A. Iyo, J. Phys. Soc. Jpn., 77:12 (2008), 124706, 7 pp., arXiv: 0810.0880.

[19] S. Shimizu, S. Tabata, H. Mukuda, Y. Kitaoka, P. M. Shirage, H. Kito, A. Iyo, Phys. Soc. Jpn., 80 (2011), 043706, 4 pp., arXiv: 1102.5282.

[20] A. N. Lavrov, L. P. Kozeeva, M. R. Trunin, V. N. Zverev, Phys. Rev. B, 79:21 (2009), 214523, $6 \mathrm{pp}$.

[21] М. Мейпл, Э. Фишер (ред.), Сверхпроводимость в тройных соединениях, Мир, М., 1985.

[22] P. D. Sacramento, J. Phys., 15:36 (2003), 6285-6300.

[23] J. V. Alvarez, Phys. Rev. Lett., 98:12 (2007), 126406.

[24] C. M. Varma, W. Webber, L. J. Randall, Phys. Rev. B, 33:2 (1986), 1015-1019.

[25] H. Tsunetsugu, M. Sigrist, K. Ueda, Rev. Mod. Phys., 69:3 (1997), 809-863.

[26] В. А. Москаленко, ТМФ, 116:3 (1998), 456-473. 
[27] В. А. Москаленко, ТМФ, 110:2 (1997), 308-322.

[28] В. В. Вальков, Д. М. Дзебисашвили, ТМФ, 157:2 (2008), 235-249.

[29] В. В. Вальков, А. О. Злотников, Изв. РАН. Сер. физ., 75:5 (2011), 682-684.

[30] Н.Н. Боголюбов, Собрание научных трудов в двенадиати томах. Статистическая механика, т. 6: Равновесная статистическая механика. 1945-1986, Наука, М., 2006.

[31] Д. Н. Зубарев, УФН, 71:1 (1960), 71-116.

[32] Н. М. Плакида, ТМФ, 5:1 (1970), 147-153.

[33] Н. М. Плакида, ТМФ, 154:1 (2008), 129-146.

[34] R. Zwanzig, Phys. Rev., 124:4 (1961), 983-992.

[35] H. Mori, Prog. Theor. Phys., 33:3 (1965), 423-455.

[36] Р. О. Зайцев, ЖЭТФ, 68:1 (1975), 207-215.

[37] Р. О. Зайцев, ЖЖЭТФ, 70:3 (1976), 1100-1111.

[38] T. Park, E.D. Bauer, J.D. Thompson, Phys. Rev. Lett., 101:17 (2008), 177002, arXiv: 0806.3308.

[39] В. В. Вальков, Д. М. Дзебисашвили, ТМФ, 162:1 (2010), 125-149.

[40] В. В. Вальков, Д. М. Дзебисашвили, ЖЭТФ, 137:2 (2010), 341-360.

[41] H. Hegger, C. Petrovich, E. G. Moshopoulou, M. F. Hundley, J. L. Sarrao, Z. Fisk, J. D. Thompson, Phys. Rev. Lett., 84:21 (2000), 4986-4989.

[42] H. Shishido, R. Settai, H. Harima, Y. Onuki, J. Phys. Soc. Jap., 74:4 (2005), 1103-1106.

[43] G. Knebel, D. Aoki, J.-P. Brison, J. Flouquet, J. Phys. Soc. Jap., 77:11 (2008), 114704, 14 pp., arXiv: 0808.3687. 